\title{
Interactive comment on "SELEN" (SELEN version 4.0): a Fortran program for solving the gravitationally and topographically self-consistent Sea Level Equation in Glacial Isostatic Adjustment modeling" by Giorgio Spada and Daniele Melini
}

Volker Klemann (Referee)

volkerk@gfz-potsdam.de

Received and published: 19 August 2019

\section{General comments}

The authors present in this manuscript an update of numerical code which enables to calculate the gravitationally consistent interaction between a surface mass load and water mass load which compensates the total mass change. Previous versions of this code were presented already on a number of workshops dealing with glacial isostatic adjustment, GIA. In contrast to these versions, the authors improved the code in a 
number of aspects which are currently discussed in GIA, and extended the portability of the code a lot. These improvements, to my point of view justify a new publication in a method and code-oriented Journal like GMD.

As I don't understand this review as a testing of the code, I will focus my review purely on the presented manuscript, and will not consider the supplement in this regard, especially for the derivation. In general, the manuscript is clearly written, the focus lies on the considered theory which the authors present in the theory section, and, as an application, the authors chose a published and established forcing for which they discuss the output in detail. Due to the fact, that the manuscript presents a methodologically oriented study, they do not discuss deviations from their results to those presented in the original publication of this forcing. From my point of view, this is reasonable strategy. As a validation of the method they refer to the benchmark study Martinec et al., 2018, which was recently published, and to which they contributed with a preliminary version of this code version.

Of course the question may arise, why the authors did not present the results for the benchmark, and discuss the addition of the rotational effect which was not considered in that benchmark this would be a nice extension to that study. If they won't do this, at least they should state, that the results of that version are identical with the current one, if rotation is switched off. Consulting that study, the SELEN code deviates a bit more than the order models when considering moving coastlines and floating ice. But this is only a suggestion, to establish more a benchmark study, than the quite complicated ICE6G vm5a applied here.

In the following, I focus on individual aspects of the presentation.

to Introduction

Printer-friendly version

P. 2, L 16: Elasticity is material law, but not a rheology.

Discussion paper

to Theory 
To present a reduced version of a derivation is every time dangerous, especially if this is not put into an appendix but a supplement which is not part of the manuscript. So, a number of questions arose, which partly come up in the following comments.

P. 4, L 7: You refer to SSM19, here you should at least specify on which principles this expression (Eq. 2) is based.

P. 4, L 10: The meaning of OF should be defined explicitly.

P. 4, L 12: You introduce here the term bedrock topography, but do not specify what it means, especially as you further down use this quantity, to derive changes in sea level.

P. 4, L 16: Here and in the following, you use the 'cal' symbol to specify variations with respect to a reference state. If so, you can of course reduce the number of equations, e.g., Eq.s 6, 11, 13 and 15 become redundant. Furthermore, you do not speficy the reference state itself.

P. 4, L 24: I don't htink that you have to refere to SSM19 to introduce the definition (7), but simply if follows from $\$ \backslash$ cal $M / \backslash$ int_e dA $\$$.

P. 5, L 2: This is somehow abrupt, and do you really need it here?

P. 5, L 4: What do you understand as a plausible surface load. At least you should guide the reader a bit more than referring to Bevis et al. 2016.

P. 5, L13: Is 'stem' used here synonymously to 'is associated'? If so, why then using a different word.

P. 5, L 5ff: How do you ensure in (Eq. 9) that you considered all terms with respect to $\$ \backslash$ cal $M \$$ ? In Eq. $5, I$ ike that $\backslash$ cal $O$ can be one of $\backslash\{-1,0,1\} \backslash$.

P. 6, L 20ff: The definition of seal level from topography is a bit tricky to understand, as you did not define topography itself properly, also sea surface is not defined her properly. So, why not motivate it from Gauss definition of the geoid as that equipotential surface to which a static ocean surface would adjust? Then, the jump from $\mathrm{N}$ to $\mathrm{r}^{\wedge} \mathrm{ss}$

Printer-friendly version

Discussion paper 
is not as large. Also at the beginning you should specify what the sea level equation expresses. Only then you can obtain its most basic form as U-N. Also the relation to the water column might help the reader to understand its relation to loading more intuitively.

P. 6, L 12: This is also known as Bruhns formula. As $\$ \backslash$ cal . $\$$ is not applied to $\$ P h i \$, I$ suggest to write this equation a bit different: $\backslash$ cal $G=\backslash$ frac $\{\backslash P h i$ - $\backslash$ Phi $\}\{$ g_0 , to remain in the used schema of formula symbols.

P. 6, L 13: One important reference in your discussion of the SLE is missing which is Martinec and Hagedoorn, 2014, who discuss in detail the SLE of a rotating body. They, for instance, distinguish between gravity and gravitational potential, where the latter only considers the surface loading, i.e. variation due to surface mass change and solid earth deformation. The gravity potential, MH14 use for gravitational potential plus rotational effects. This is in accordance to Helmut Moritz, 1990, The figure of the earth. So I would use gravity potential instead of geopotential.

P. 6, L 14: The meaning of $c$ only depending on $\$ \mathrm{t} \$$, can easily be explained simply by the fact, that the displacement of the reference potential surface, N_0, does not represent the potential level to which the water surface will adjust. This is not only due to mass conservation but also due to the changes in the ocean basin. So, it is a bit more complicate.

P. 7, L 10: Here and in the following, $\$ O \$$ is dangerous to use, as it is easily mistaken for the reference state 0 .

P. 7, L 12: From type setting it is nicer to use text abbrevations in formulas with an unslanted font, e.g., $\$\{\backslash \text { cal } S\}^{\wedge} \backslash$ text\{ave $\$$.

P. 7, 14: For the definition of the different sea-levels see below. For me, equivalent is more a renormed quantity, but here you are using it with respect to a model dependent quantity, depending on the current ocean area $A^{\wedge} \mathrm{o}(\backslash$ gamma, $\mathrm{t}$ ) and \mu (gamma, t).

Printer-friendly version

Discussion paper 
Both terms depend on the $\$ \backslash$ cal $S \$$ and $\$ \backslash$ cal $L^{\wedge}\{[a b c] \$$.

P. 7, L 8ff: This derivation is quite interesting, and I have needed some time to understand this alternative expression, especially why in Eq. 33 \$T_0\$ appears instead of \$T\$. May be, a specification what 'using for \$c\$ the expression found in SSM19' does mean, might help, as $c$ does not appear anymore. The interesting aspect is that this form is independent of the syphoning, which dominates $\langle\mathrm{S}\rangle^{\wedge} \mathrm{e}$, and is put into $<\mathrm{G}-\mathrm{U}\rangle^{\wedge} \mathrm{O}$. May be this might help to understand Table 4 a bit more. So I guess the expression, $\$-\left\langle\mathrm{G}-\mathrm{U}^{\wedge} \mathrm{O}+\mathrm{S}^{\wedge}\right.$ ave $=\mathrm{c} \$$ is missing.

P. $8 \mathrm{~L}$ 3: Wikipedia defines the 'eustatic change' as the alteration of sea level due to changes in volume of water or ice, and also due to changes in ocean topography, which goes back to Suess. The article to my point of view is based also on Rovere et al. (2016, https://doi.org/10.1007/s40641-016-0045-7), that is, also steric changes are considered. I would call Eq. 36, the equivalent sea level, as you also do at P. 10, L. 14ff, expressing an ocean equivalent of the ice, which is simply a renormation of water mass expressed as water height. S^ave, I like. Your $S^{\wedge}$ eus, I would call $\backslash$ bar $\{\backslash$ cal S\}^ice, what is the equivalent of ice at current state. Your $S^{\wedge}$ equ is a help quantity, I would keep its definition. FC76 phrase Eq. (36) to be the eustatic sea level although the ocean basin might slightly change. The authors are aware of the fact and "tried to avoid [...] the word (eustasy) has received so many qualifications since it was introduced by Suess". So, I would suggest not to use eustasy at all. It is a deprecated definition of sea level and mainly leads to misunderstanding.

P. 8, L 17: 'essentially' is a bit enigmatic, do you mean terms of $d / 0=2 / 0$ and $0 / 0$ can be neglected as they are too small? See also MH2014.

P. 8. L 7: From here to the end of this section, you discuss the numerical implementation into SELEN. As the whole article is about SELEN, I would expect a bit more detail, how the equations are solved also with respect to the iterations you discuss in the following. So, this part I suggest to extend.

Printer-friendly version

Discussion paper 


\section{to $A$ test run of SELEN}

In the introduction to this section you should mention that you also consider changes in rotation in addition to $\mathrm{N}, \mathrm{U}$ and $\mathrm{S}$.

P. 10, L. 23: Why do you use an interpolation. To go from a fine to a coarse grid by interpolation is everytime a bit dangerous. I would expect here a filtering or a binning algorithm.

P. 11, L 10: 'Not agreed results on ve. Love numbers'. Do you have a reference for this statement, and why this is an argument to neglect compressibility?

P. 11, L 15: I would simply start the sentence: 'Numerical values of [. . . in Table 3 and its caption, respectively', to avoid using two times reference, and the reader to search for the tidal love numbers in the table rows.

P. 11, L 17 and P. 14, L 9: For the captions use 'Glacial isostatic adjustment in the past' and '[... . at present day' or alternatively 'Paleo glacial isostatic adjustment' and 'Present-day [...]'.

P 11, P 27: I would in one sentence introduce the three configurations you discuss in the following.

P. 13, L 2: Why do you mention the small region around Patagonia with a reference only. Why not citing Klemann et al. (2015, DOI 10.1007/s41063-015-0004-x) for the East Siberian shelf or Lambeck for the Sunda Shelf. Furthermore, the light blue areas may represent locations covered with grounded ice, but also floating ice might be present there. The ice extent is not shown in Fig. 5.

P. 13, L 20. As stated before the MH14 paper, is in my point of view an important contribution to the discussion of rotation in GIA.

Printer-friendly version

Discussion paper

P. 13, L 25: 'since' used twice.

P. 14, L 8: For such statement, you should at least relate the order to that of other 
transient processes observed or proposed for rotational variations.

P. 14, L 11: Why 'shall', although you consider it.

P. 14, L 15: Here you define $\$ \backslash$ cal $S \$$ as relative sea level, I suggest you to use this definition from the beginning, what also would clearify what the SLE is solving for.

P. 14, L 16: would repeat 'frame' also before b, c and d.

P. 15, L 4: This is only clear from the fact that the syphoning effect is inside the definition of $\$ \backslash$ gamma $\$$-depending part, otherwise the reader would wonder.

P. 15, L 8: Instead of 'decontamination', geodesists speak about 'correction for' an effect.

P 15, L 20: 'then' to 'them'.

P. 16, L 23-25: This is an interesting aspect. Can you give a formula to indicate which term has to neglected as direct rotational effect?

P. 16, L 32: Is this the degree variance, and why not presenting the equation here?

to Conclusions

P. 16, L 23-25: This is an interesting aspect. Can you give a formula to indicate which term has to neglected as direct rotational effect?

P. 16, L 32: Is this the degree variance, and why not presenting the equation here?

P. 17, L 12: replace 'runs' by 'run'.

P. 17, L 15: Not sure, if one can speak about 'physical realism'.

to figures and tables

Printer-friendly version

Figure 1: In this color scheme, the dark ice thicknesses make it difficult to identify the coast lines. In this caption and in the further ones, I would not state which plotting command you used. 
Figure 2: I would skip this figure.

Table 2: 'Density, [...] values specifying the parameters of the homogeneous layers defining the adopted [...]'. 'Some spectral [...]' I would skip this sentence, as it does not contribute to the table content.

Table 3: The PMTF is not defined in the manuscript. Without it, the $\mathrm{A}^{\wedge}[\mathrm{es}]$ and [hkl] ${ }^{\wedge} \mathrm{T}$ are not necessary. In this context it would be interesting, if you need also viscoelastic tidal love numbers.

Figure 8: Can you specify the extreme values reached in the respective plots?

Table 4: I would skip the integrals over the whole earth, as these are clear from the definition of $U$ and $G$.

Interactive comment on Geosci. Model Dev. Discuss., https://doi.org/10.5194/gmd-2019-183, 2019. 